\title{
Lübeck Beech Forest Biodiversity Plots
}

\author{
Christian Dolnik, Leonid Rasran \& Kati Vogt
}

\begin{abstract}
The database Lübeck Beech Forest Biodiversity Plots (GIVD ID EU-DE-031) was established in 2007 at the Ecology Centre of Kiel University in co-operation with the Lübeck forest administration in the framework of a beech forest biodiversity project supported by the German Environmental Foundation (DBU). Study objects are temperate Central European lowland beech forest of managed and of unmanaged forest stands (since 10 and 50 years). The database contains 86 series of nested plots from 0.0001 to 1,000 $\mathrm{m}^{2}$ and geobotanical relevés for the 400 and $1,000 \mathrm{~m}^{2}$ plot size. A census was taken for vascular plants, bryophytes and lichens, including epiphytes.
\end{abstract}

Keywords: epiphyte; Fagus sylvatica; Germany; nested plot.

GIVD Database ID: EU-DE-031

Last update: $2012-05-09$

\section{Lübeck Beech Forest Biodiversity Plots}

Scope: The database contains 86 geobotanical relevés of Central European beech forest of managed and of unmanaged forest stands (since 10 and $50 \mathrm{yr}$.).

Status: finished Period: 2007-2008

Database manager(s): Christian Dolnik (cdolnik@ecology.uni-kiel.de)

Owner: Ecology Centre Kiel

Web address: [NA]

Availability: according to a specific agreement

Database format(s): MS Access

Publication: [NA]

Plot type(s): nested plots

Non-overlapping plots: 86

Total plot observations: 172

Countries: DE: $100.0 \%$

Forest: [NA] - Non-forest: [NA]

Guilds: all vascular plants: $100 \%$; bryophytes (terricolous or aquatic): 100\%; lichens (terricolous or aquatic): 100\%; non-terricolous taxa (epiphytic, saxicolous, lignicolous): $100 \%$

Environmental data: [NA]

Performance measure(s): cover: $100 \%$

Geographic localisation: GPS coordinates (precision 25 m or less): $100 \%$

Sampling periods: $2000-2009: 100.0 \%$

Information as of 2012-07-12; further details and future updates available from http://www.givd.info/ID/EU-DE-031

Christian Dolnik* (cdolnik@ecology.uni-kiel.de), Leonid Rasran (lrasran@ecology.uni-kiel.de), Kati Vogt (kvogt@ecology.unikiel.de)

Ecology Centre Kiel, University of Kiel, Olshausenstr. 40, 24098 Kiel, GERMANY

*Corresponding author 